\title{
Prophylactic mastectomy
}

\author{
M. P. Osborne \\ Strang Cancer Prevention Center, New York, USA.
}

Abstract Women with increased risk of breast cancer have the treatment options of chemoprevention, surveillance or prophylactic mastectomy (PM). This article will focus on PM. PM should only be undergone after a detailed risk assessment and consultation with the patient's entire medical team, including a psychologist. PM will either be bilateral or contralateral. Bilateral PM is usually recommended to women who have a genetic predisposition for breast cancer. Research has shown that PM was $98.6 \%$ effective in preventing breast cancer in BRCA1 or BRCA2 mutation carriers. Contralateral PM (CPM) is a treatment option not often recommended to women who are being treated for breast cancer. While research has shown that CPM can offer a 95\% risk reduction to contralateral breast cancer recurrence, the low risk of developing a contralateral breast cancer in the first place $(0.6 \%)$ means that CPM would actually offer little benefit to most patients. However, it should be noted that the efficacy of PM for patients with invasive lobular carcinoma is controversial and further research is needed. The optimal surgical technique for removing the most breast tissue is total mastectomy, while new techniques of 'skin sparing' mastectomy will allow a better cosmetic outcome following reconstruction.

Keywords: Breast cancer; Prophylactic mastectomy; Bilateral prophylactic mastectomy; Contralateral prophylactic mastectomy; BRCA1 and BRCA2 mutation

\section{Introduction}

The options for women at increased risk of breast cancer are surveillance, chemoprevention and prophylactic mastectomy (PM) [1].

Increased risk includes:

- A family history consistent with an inherited autosomal dominant mutation on the BRCA1 or 2 or unknown genes. This type of family history is characterized by, breast and/or ovarian cancer in multiple affected relatives, young age of onset $(<50$ years), bilaterality and vertical transmission between generations.

Correspondence to: Michael P. Osborne MD, MS, FRCS, FACS, Strang Cancer Prevention Center, 428 East 72nd Street, Suite 600, New York, NY 10021, USA E-mail: osborne@strang.org; Tel: +1 212746 6629; Fax: +1212 3961244

Publication date 25/02/05 BCO/110/2002/FO
- The presence of atypical ductal or lobular epithelial hyperplasia [2] or lobular carcinoma in situ (lobular neoplasia) in breast biopsies [3]. These findings do not usually have any clinical or radiological manifestations and are found incidentally in $\approx 5 \%$ of benign breast biopsies.

- Age-related risk is not sufficient to justify any intervention beyond physical examination and annual screening mammography.

- A personal history of breast cancer indicates an increased risk of contralateral breast cancer.

The decision to undergo PM requires detailed assessment and counselling by the surgeon, reconstructive surgeon, as well as genetic and psychological counselling. The procedure is radical, irreversible and should not be decided on without comprehensive counselling and considerable thought by the patient. 


\section{Bilateral PM}

A survey of plastic surgeons in the US showed that bilateral prophylactic subcutaneous mastectomy was effective in preventing breast cancer. Out of 1500 patients followed for an average of 9 years, six breast cancers were reported. However, $30 \%$ were lost to follow up [4]. A comprehensive retrospective study of 214 women undergoing PM at the Mayo Clinic, with a median follow up of 14 years, showed that PM was $98.6 \%$ effective when compared with their sisters who did not undergo PM [5]. Subsequent analysis of a small subset of 26 women who had a BRCA1 or 2 mutations and underwent PM showed that none developed breast cancer after a median follow up of 13 years [6].

In a prospective study of 139 women in Holland, with either BRCA1 or 2 mutations, or a family history consistent with an inherited predisposition to breast cancer, none developed breast cancer after PM, compared to eight in the group who underwent surveillance and did not have PM [7]. This represents a $17 \%$ incidence of cancer in the surveillance group and $100 \%$ efficacy of PM after a short mean follow up of 3 years.

\section{Contralateral PM}

Contralateral PM (CPM) is not routinely recommended to women who are being treated for breast cancer. The contralateral breast cancer rate is $0.6 \%$ per year and surveillance is recommended [8]. The contralateral risk for patients with invasive lobular carcinoma is controversial.

A study of 745 patients at the Mayo Clinic underwent CPM and eight subsequently developed contralateral breast cancer representing a $\approx 95 \%$ risk reduction at a median follow up of 10 years [6]. In a case-control study of 64 women who underwent CPM were compared to 182 control women who did not, at a mean follow up of 15 years, none of the women had subsequently developed contralateral breast cancer compared to 36 in the control group [9]. A significant increase in disease-free survival was observed for those undergoing CPM $(P=0.01)$ and the improvement in overall survival at 15 years was $68 \%$ for those undergoing CPM compared to $48 \%$ for controls, but was not statistically significant $(P=0.26)$. In patients with high risk of metastatic disease CPM will not contribute much to overall survival, however in patients with early-detected Stage I breast cancer CPM may contribute more but no data are available to support this hypothesis.

\section{Surgical technique}

Anatomic studies have shown that even the most radical surgery cannot remove all the breast tissue
[10]. Subcutaneous mastectomy through an inframammary incision will leave residual breast tissue in the subareolar and axillary tail areas. The optimal surgical approach is total mastectomy, which removes the nipple/areola complex and provides better exposure for a more complete procedure. New approaches using 'skin-sparing' techniques provide a better cosmetic outcome after reconstruction [11]. Immediate reconstruction with either saline implants or autologous tissue should be discussed at the time PM is discussed. Sentinel node biopsy at the time of PM can be considered because $\approx 5 \%$ of patients undergoing PM have an occult breast cancer present and the need for a subsequent axillary dissection may be obviated by this approach.

\section{Psychological issues}

Many patients considering PM overestimate their risk of breast cancer [12]. This is particularly true of patients who have not been genetically tested [13]. Regrets after PM have been described in $\approx 5 \%$ of women in a self-selected national registry [14]. In a study, from the Mayo Clinic only $\approx 30 \%$ were entirely satisfied with their decision [15], however this emphasizes the need for very careful preoperative counselling in order not to overly elevate expectations.

\section{Conclusion}

PM is an option for women at high risk of breast cancer because of an inherited predisposition, specific pathologic findings after a breast biopsy or a personal history of breast cancer.

Comprehensive pre-procedure counselling is required and potential aesthetic outcomes should be discussed so as to not overly elevate expectations.

\section{References}

1. Osborne MP. Prophylactic mastectomy. Semin Breast Disease 2003; 6: 34-40.

2. Osborne MP, Borgen PI. Atypical ductal and lobular hyperplasia and breast cancer risk. Surg Oncol Clin North Am 1993; 2: 1-11.

3. Osborne MP, Hoda S. Current management of lobular carcinoma in situ of the breast. Oncology 1994; 8: 45-49.

4. Pennisi VR, Capozzi A. Subcutaneous mastectomy data: final statistical analysis. Aesthetic Plast Surg 1989; 13: 15-21.

5. Hartmann LC, Schaid DJ, Woods JE, et al. Efficacy of bilateral prophylactic mastectomy in women with a family history of breast cancer. New Eng J Med 1999; 340: 77-84.

6. Hartmann LC, Sellers TA, Schaid DJ, et al. Efficacy of bilateral prophylactic mastectomy in BRCA1 and BRCA2 gene mutation carriers. J Natl Cancer Inst 2001; 93 : 1633-1637. 
7. Meijers-Heijboer $\mathrm{H}$, van Gell $\mathrm{B}$, van Patten $\mathrm{WL}$, et al. Breast cancer after prophylactic bilateral mastectomy in women with a BRCA1 or BRCA2 mutation. New Eng $J$ Med 2001; 345: 159-164.

8. Adair F, Berg J, Jonbert L, et al. Long-term follow-up of breast cancer patients. The 30 years report. Cancer 1974; 33: 1145-1150.

9. Peralta EA, Ellenhorn JD, Wagmen LD, et al. Contralateral prophylactic mastectomy improves the outcome of selected patients undergoing mastectomy for breast cancer. Am J Surg Oncol 2000; 180: 439-445.

10. Hicken NF. Mastectomy. Clinical pathologic study demonstrating why most mastectomies result in incomplete removal of the mammary gland. Arch Surg 1940; 40: 6-14.

11. Simmons RM, Fish SK, Gayle L, LaTrenta GS, Swistel A, Christos P, Osborne MP. Local and distant recurrence rates in skin-sparing mastectomies compared with non-skin-sparing mastectomies. Ann Surg Oncol 1999; 6: 676-681.

12. Meiser $B$, Butow $P$, Friedlander $M$, et al. Intention to undergo prophylactic bilateral mastectomy in women at increased risk of developing hereditary breast cancer. J Clin Oncol 2000; 18: 2250-2257.

13. Metcalfe KA, Narod SA. Breast cancer risk perception among women with contralateral prophylactic mastectomies. J Natl Cancer Inst 2002; 94: 1564-1569.

14. Montgomery LL, Tran KN, Heelan ME, et al. Issues of regret in women with contralateral prophylactic mastectomies. Ann Surg Oncol 1999; 6: 546-552.

15. Frost MH, Schaid DJ, Sellers TA, et al. Long-term satisfaction and psychological and social function following bilateral prophylactic mastectomy. J Am Med Assoc 2000; 284: 319-324. 